\title{
Xenotropic and polytropic retrovirus receptor 1 (XPR1) promotes progression of tongue squamous cell carcinoma (TSCC) via activation of NF-KB signaling
}

Wei-chao Chen ${ }^{1,2,3+}$, Qiu-li Li ${ }^{1,2,3+}$, Qimei Pan ${ }^{4 \dagger}$, Hua-yong Zhang ${ }^{1,2,3}$, Xiao-yan Fu' ${ }^{1,2,3}$, Fan Yao ${ }^{1,2,3}$, Jian-ning Wang ${ }^{5^{*}}$ and An-kui Yang ${ }^{1,2,3^{*}}$

\begin{abstract}
Background: Xenotropic and polytropic retrovirus receptor 1 (XPR1), a previously identified cellular receptor for several murine leukemia viruses, plays a role in many pathophysiological processes. However, the role of XPR1 in human cancers has not yet been characterized.

Methods: Real-time PCR and western blotting assay were used to measure the expression of XPR1 in tongue squamous cell carcinoma (TSCC) tissues. Expression of XPR1 and p65 in clinical specimens was analyzed using immunohistochemical assay. The function of XPR1 on progression of TSCC was explored using in vitro and in vivo experiments. The molecular mechanism by which XPR1 helps to cancer progression was investigated by luciferase reporter activity, ELISA, PKA activity assay, immunofluorescence, western blotting and qPCR assay.

Results: Herein, we find that XPR1 is markedly upregulated in TSCC tissues compared to normal tongue tissues. High expression of XPR1 significantly correlates with the malignant features and poor patient survival in TSCC. Ectopic expression of XPR1 increases, while silencing of XPR1 reduces the proliferation, invasion and anti-apoptosis capacities of TSCC cells. Importantly, silencing of XPR1 effectively inhibits the tumorigenecity of TSCC cells. Moreover, we identified that XPR1 increased the concentration of intracellular CAMP and activated PKA. Thus, XPR1 promoted phosphorylation and activation of NF-KB signaling, which is required for XPR1-mediated oncogenic roles and significantly correlates with XPR1 expression in clinical specimens.
\end{abstract}

Conclusions: These findings uncover a critical role of XPR1 in TSCC progression via activation of NF-KB, and suggest that XPR1 might be a potential prognostic marker or therapeutic target.

Keywords: XPR1, TSCC, NF-KB signaling, Prognostic marker, Therapeutic target

\footnotetext{
* Correspondence: wangjn@mail.sysu.edu.cn; akyang_123@sina.com

${ }^{+}$Wei-chao Chen, Qiu-li Li and Qimei Pan contributed equally to this work. ${ }^{5}$ Department of Oral and Maxillofacial Surgery, Guanghua School of Stomatology, Hospital of Stomatology, Institute of Stomatological Research, Sun Yat-sen University, Guangdong Provincial Key Laboratory of

Stomatology, Guangzhou, Guangdong Province 510055, People's Republic of

China

'Department of Head and Neck, Sun Yat-sen University Cancer Center,

Guangzhou, Guangdong 510060, People's Republic of China

Full list of author information is available at the end of the article
}

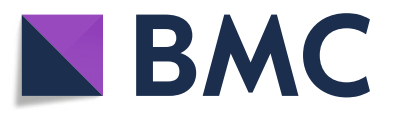

(c) The Author(s). 2019 Open Access This article is distributed under the terms of the Creative Commons Attribution 4.0 International License (http://creativecommons.org/licenses/by/4.0/), which permits unrestricted use, distribution, and reproduction in any medium, provided you give appropriate credit to the original author(s) and the source, provide a link to the Creative Commons license, and indicate if changes were made. The Creative Commons Public Domain Dedication waiver (http://creativecommons.org/publicdomain/zero/1.0/) applies to the data made available in this article, unless otherwise stated. 


\section{Background}

Oral squamous cell carcinoma (OSCC) is one of the most common malignant tumors in the head and neck, and it is well-known for its highly proliferative and invasive nature. Tongue squamous cell carcinoma (TSCC) is the most common type of OSCC [1, 2]. Extensive surgery combined with chemotherapy and radiotherapy has been used as the primary clinical treatments for OSCC patients [3-5]. Despite considerable advances in diagnostics and therapies for this disease, the overall 5-year survival rate for OSCC patients stuck around 50\% without marked improvement, because of the high risks of developing secondary or recurrent tumors in surrounding area [6-9]. Hence, this underscores the need for identifying the key molecular alterations that contribute to OSCC progression in order to identify effective therapeutic targets for patients.

Xenotropic and polytropic retrovirus receptor (XPR1) is a 696-amino acid protein with multiple transmembrane-spanning domains. It was first identified as a specific cell-surface receptor for xenotropic and polytropic classes of murine leukemia viruses (MLV) [10-12]. XPR1 has also been reported to mediate $\mathrm{G}$ protein recruitment and play a role in G-protein-coupled signal transduction [13-15]. Based on its homology to proteins involved in the regulation of phosphate transport, including PHO1, PHO90, and PHO91 [1618], XPR1 has also been identified as an inorganic phosphate exporter in human cells [19].

Notably, recent studies suggest that there is a close relationship between levels of XPR1 expression and certain pathophysiological processes. For instance, Sharmaet et al. reported that activation of the receptor-activated NF- $\kappa B$ ligand (RANKL)-receptor activated NF- $\mathrm{KB}$ (RANK) signaling pathway in murine osteoclasts leads to XPR1 upregulation, which may suggest that XPR1 plays a role during bone resorption. This group also detected a differential distribution of XPR1, which is present in the cytoplasm of mononuclear osteoclast precursors and translocates to the membranes of mature multinucleated osteoclasts, suggesting that XPR1 has a role in differentiation in these cells [20]. Several studies have found that XPR1, which is demonstrated to interact with Beta-type platelet-derived growth factor receptor (PDGFRB) and play a fundamental role in the maintenance of the cellular phosphate balance in the brain, is a pathogenic gene involved in primary familial brain calcification (PFBC) [21-23]. Research by Hueso found that XPR1, a regulator of macrophage development, is upregulated in human atherosclerotic plaques during disease progression [24]. However, the clinical significance and biological roles of XPR1 in cancer progression remain largely unknown.

In the current study, we report that XPR1 expression is substantially increased in TSCC tissues and positively correlates with the clinical malignant features of TSCC as well as poor patient prognosis. Overexpression of XPR1 might promote TSCC cell proliferation, invasion and resistance to chemotherapy both in vitro and in vivo. Moreover, we show that XPR1 activates the NF- $k B$ signaling pathway via upregulation of cAMP and subsequent PKA activation in TSCC. Collectively, our findings suggest that XPR1 plays a critical oncogenic role in TSCC progression via activation of NF- $\mathrm{kB}$ signaling, providing potential prognostic marker and therapeutic target against the disease.

\section{Patient specimen information}

We conducted IHC staining on a total of 128 archived TSCC specimens, which were histopathologically diagnosed at the Sun Yat-sen University Cancer Center from 2001 to 2009. Patients' consent and approval were obtained from the Institutional Research Ethics Committee prior to use. Information of specimens is summarized in Table S1. Eight freshly collected TSCC tissues and matched adjacent noncancerous tissues were stored in liquid nitrogen until use.

\section{Immunohistochemistry (IHC)}

We performed IHC staining on the 128 paraffin-embedded TSCC tissue sections using anti-XPR1 (Sigma, HPA016557, 1:50) and anti-p65 (CST, \#8242, 1:50) antibodies. In brief, paraffin-embedded specimens were cut into $4-\mu \mathrm{m}$ sections and baked at $65^{\circ} \mathrm{C}$ for $30 \mathrm{~min}$. The sections were deparaffinized with xylenes and rehydrated. Sections were then submerged into EDTA antigenic retrieval buffer and microwaved for antigenic retrieval. Samples were treated with 3\% hydrogen peroxide in methanol to quench the endogenous peroxidase activity, followed by incubation with $1 \%$ bovine serum albumin to block nonspecific binding, and then incubated with primary antibodies overnight at $4^{\circ}$ C. After washing, the tissue sections were treated with biotinylated anti-rabbit secondary antibody, followed by further incubation with streptavidin-horseradish peroxidase complex (Zsbio, BJ, China). Finally, the sections were immersed in 3-amino-9-ethyl carbazole and counterstained with $10 \%$ Mayer's hematoxylin, dehydrated, and mounted in Crystal Mount. The XPR1 staining was graded with four scores, strong +3 , moderate +2 , weak +1 , and negative 0 . Specimens with scores $+3,+2$ were defined as high expression; while the others scored as +1 or 0 were low expression. On the other hand, specimens with $>10 \%$ nuclear p 65 expression were defined as nuclear p65-positive, and specimens with $\leq 10 \%$ nuclear p65 expression were p65-cytoplasmic.

\section{Vectors, retroviral infection, and transfection}

PCR-amplified human XPR1 coding sequence was cloned into the pLVX-IRES-puro vector. To silence 
endogenous XPR1, two RNAi oligonucleotides were cloned into the pSuper-retro-puro vector. shRNA sequences were as followed: shRNA\#1: GCGAUUUGU GUGGAACUUCUU; shRNA\#2: CGUGACACUAAGGU AUUGAUA. Transfection was performed using the Lipofectamine 2000 reagent (Invitrogen, Carlsbad, CA, USA) according to the manufacturer's instructions. Stable cell lines expressing XPR1 or XPR1-shRNAs were selected for 10 days with $0.5 \mu \mathrm{g} / \mathrm{ml}$ puromycin. The cell lysates prepared from the pooled population of cells in sample buffer were fractionated using SDS-PAGE to quantify XPR1 protein levels.

\section{Western blotting analysis}

Western blotting was performed using anti-XPR1 (Sigma, Saint Louis, MO, USA), anti-p65, and anti-p84 antibodies (Cell Signaling, Danvers, MA, USA). The membranes were stripped and re-probed with an anti- $\alpha$-tubulin antibody (Sigma, Saint Louis, MO, USA) as a loading control.

\section{Colony formation assay}

One thousand cells were plated in 6-well plate and cultured for 10 days. The colonies were fixed stained with $10 \%$ formaldehyde for $5 \mathrm{~min}$ and stained with $1.0 \%$ crystal violet for $30 \mathrm{~s}$. Colonie numbers were counted and shown as mean $\pm \mathrm{SD}$.

\section{Transwell penetration assay}

$1 \times 10^{4}$ Cells were plated into the upper chamber of polycarbonate filters coated with Matrigel (BD Biosciences, San Jose, CA, USA) and cultured at $37^{\circ} \mathrm{C}$ for 24 h. Invading cells were fixed in $1 \%$ paraformaldehyde, stained with crystal violet and counted in five random fields per well. The data was shown as mean \pm SD.

\section{Annexin V-FITC/propidium iodide (PI)-stained assay}

Cells were trypsinized, washed in ice-cold PBS and centrifuged at $1000 \mathrm{~g}$ for $5 \mathrm{~min}$. The pellet was resuspended in binding buffer at a density of $1.0 \times 10^{6} \mathrm{cells} / \mathrm{ml}$. Following this, $100 \mu$ l of the sample solution was incubated with $5 \mu \mathrm{l}$ of FITC-conjugated Annexin V and $5 \mu \mathrm{l}$ of PI for $15 \mathrm{~min}$ at $37^{\circ} \mathrm{C}$ in dark. Subsequently, $400 \mu \mathrm{l}$ of binding buffer was added to each sample and the samples were analyzed by flow cytometry. Apoptotic cells was caculated by the portion of Annexin V-positive cells.

\section{Xenograft tumor model}

All animal experimental procedures were approved by the Institutional Animal Care and Use Committee of Sun Yat-sen University Cancer Center. Briefly, BALB/ c-nu mice (5-6 weeks, 18-20 g, $n=6$ ) were inoculated subcutaneously with $5 \times 10^{6}$ SCC25 vector or XPR1-knockdown cells in the dorsal flanks. Tumor volumes were determined every week. Tumor volume was calculated using the eq. $\left(\mathrm{L}^{*} \mathrm{~W}^{2}\right) / 2$. After 7 weeks, the mice were euthanized and imaged, and the tumors were excised and weighed. Serial $6.0-\mu \mathrm{m}$ sections were cut and subjected to immunohistochemical staining of Ki67 and TUNEL. Proliferation index was quantified by counting the proportion of Ki67-positive cells, while apoptotic index was quantified by the percentage of TUNEL-staining cells.

\section{Luciferase reporter assays}

Cells $\left(5 \times 10^{4}\right)$ were seeded in triplicate in 24-well plates and cultured for $24 \mathrm{~h}$. One hundred nanograms of luciferase reporters plus $5 \mathrm{ng}$ of pRL-TK Renilla plasmid (Promega, Madison, WI) were transfected into the indicated cells using Lipofectamine 2000 reagent (Invitrogen Co., Carlsbad, CA) according to the manufacturer's recommendations. Luciferase and Renilla signals were measured $36 \mathrm{~h}$ after transfection using the Dual Luciferase Reporter Assay Kit (Promega, Madison, WI).

\section{ELISA analysis of CAMP and PKA activity assay}

The concentration of cAMP and activity of PKA in the cell lysates of TSCC cell lines were measured using a cAMP Assay Kit (Abcam, ab65355) and PKA Kinase Activity Assay Kit (Abcam, ab139435) respectively, according to the manufacturer's protocol.

\section{Immunofluorescence}

Cells $\left(5 \times 10^{4}\right)$ were plated on coverslips to culture for $24 \mathrm{~h}$. Cells were washed three times with PBS and treated with $1 \%$ Triton X-100. Next, cells were stained with anti-p65 antibody (Cell Signaling Technology, 1:200) for $2 \mathrm{~h}$ at $4{ }^{\circ} \mathrm{C}$. After washing three times with PBS, the cells were incubated with FITC-conjugated goat anti-mouse antibody (Cell Signaling Technology, 1:100) at $37^{\circ} \mathrm{C}$ for $1 \mathrm{~h}$. Cells were counterstained with DAPI (Sigma-Aldrich) to visualize the nuclei.

\section{Chemical reagents}

NF- $\mathrm{kB}$ inhibitor quinazoline (QNZ) was purchased from Selleck Chemicals and used at a concentration of $10 \mathrm{nM}$.

\section{Statistical analysis}

Statistical tests for data analysis included the log-rank test, $\mathrm{X}^{2}$ test and Student's two-tailed $t$-test. Multivariate statistical analysis was performed using a Cox regression model. Statistical analyses were performed using the SPSS11.0 statistical software package. Data represent the mean \pm SD. $P$ values of 0.05 or less were considered statistically significant. 


\section{Results}

XPR1 overexpression correlates with progression and poor prognosis in TSCC

To investigate the role of XPR1 in cancer, we first analyze its expression. Interestingly, analysis with the Cancer Genome Atlas (TCGA) Head and Neck Cancer (HNSCC) dataset suggested that XPR1 was robustly increased in tumor samples compared to normal tissues (Additional file 1: Figure S1A). More importantly, high expression of XPR1 significantly predicted poorer overall survival in patients with HNSC in TCGA data, as indicated by the human protein atlas program (https://www.proteinatlas.org/, Additional file 1: Figure S1B). These data suggest that XPR1 might play an important role in the progression of head and neck cancer.

We then validated the upregulation of XPR1 in TSCC, a common subtype of head and neck cancer. As shown in Fig. 1a and b, both mRNA and protein levels of XPR1 were robustly upregulated in 8 paired human TSCC tissues $(\mathrm{T})$ compared with the matched adjacent noncancerous tissues (ANT). Furthermore, we assessed the expression status of XPR1 by immunohistochemistry (IHC) staining in 128 archived TSCC specimens and 4 normal tongue tissues (Additional file 1: Table S1). XPR1 was undetected in 4 normal tongue tissues (Fig. 1c). IHC analysis revealed that 27 contained strong $(+3)$, 40 contained moderate $(+2)$, and 52 had weak $(+1)$ expression levels of XPR1, while 9 negatively (0) expressed XPR1 (Fig. 1c). Correlation analysis showed that the distribution of XPR1 staining was positively and significantly associated with $\mathrm{T}(P=0.023)$ and $\mathrm{N}(P=0.039)$ classifications in TSCC patients (Fig. 1d). The specimens with score $+3,+2$ were defined as XPR1-high, while the others were XPR1-low (Additional file 1: Figure S1C). Importantly, Kaplan-Meier survival curves and log-rank tests revealed that patients with high expression of XPR1 significantly had higher risk and poorer overall survival $(P=0.002$, hazard ratio $(95 \% \mathrm{CI})=3.163(1.541-$ 6.493), Fig. 1e). Moreover, multivariate Cox regression analysis indicated that high expression of XPR1, patient age and $\mathrm{T}$ classification were each recognized as independent prognostic factors for the overall survival in TSCC (Additional file 1: Table S2). These results suggest that overexpression of XPR1 might contribute to TSCC progression, leading to poor clinical outcome.

\section{XPR1 promotes aggressiveness of TSCC cells in vitro}

To investigate the biological role of XPR1, we first stably expressed XPR1 in TSCC cell lines SCC25 and CAL-27 by lentivirus infection (Fig. 2a). MTT assays revealed that ectopic expression of XPR1 promoted significant increase in viability of both SCC25 and CAL-27 cells (Fig. $2 \mathrm{~b})$, and these results were further confirmed by the colony formation assays (Fig. 2c). Moreover, transwell penetration assays showed that XPR1 strongly increased the invasive capacity of TSCC cells (Fig. 2d). In addition, XPR1 effectively reduced cisplatin-induced cell apoptosis, suggesting that XPR1 also contibuted to the resistance to chemotherapy in TSCC (Fig. 2e).

On the other hand, silencing of XPR1 significantly reduced the proliferation, invasion and chemoresistance capacities of TSCC cells (Fig. 3a-e). Notably, alterations of XPR1 expression had no significant effects on basal apoptosis rate of TSCC cells (Additional file 1: Figure S2A and B). Collectively, these results suggest that XPR1 promotes aggressiveness of TSCC cells in vitro.

\section{Silencing of XPR1 inhibits tumorigenecity of TSCC cells in vivo}

We next investigated the role of XPR1 in the tumorigenicity of TSCC cells. $5 \times 10^{6}$ control and shRNAs-mediated XPR1 knockdown SCC-25 were injected subcutaneously into Balb/c nude mice and inoculated for 7 weeks. Strikingly, tumors formed by XPR1 knockdown SCC25 cells grew much more slowly than scramble-transduced control SCC25 cells as indicated by the tumor volumes and weights (Fig. $4 \mathrm{a}-\mathrm{c}$ ). Consistently, IHC staining of $\mathrm{Ki} 67$ revealed that silencing of XPR1 inhibited cell proliferation of TSCC cells, but increased the percentage of TUNEL-stained cells in xenografts (Fig. 4d and e). These findings indicate that silencing of XPR1 reduced the tumorigenecity of TSCC cells, and suggest that XPR1 might be a therapuetic target.

\section{XPR1 activates NF-KB signaling}

To explore the mechanism for the oncogenic effects of XPR1 in TSCC, we conducted assays of luciferase reporters of serveral typical oncogenic pathways including STAT3, NF-kB, FOXO1 and Wnt/ $\beta$-catenin. Interestingly, the luciferase reporter assays revealed that XPR1 markedly increased the activity of NF-kB, but showed little effects on other signaling pathways (Fig. 5a). Consistently, silencing of XPR1 significantly inhibited the luciferase activity of NF- $\mathrm{kB}$ in SCC-25 and CAL-27 cells (Fig. 5b). Notably, XPR1 was found as a G-protein-coupled receptor to stimulate cAMP production [1], and cAMP activates PKA kinase, which phosphorylates p65 at ser276 to promote nuclear translocation and transcriptional activation of NF- $\mathrm{kB}$. Strikingly, our results indicated that overexpression of XPR1 increased, while silencing of XPR1 reduced the concentration of cAMP and activity of PKA in SCC-25 and CAL-27 cell lines (Fig. 5c and d). XPR1 increased the ser276 phosphorylation of p65 (Fig. 5e). Fluorescence immunostaining and cellular fractionation showed that overexpression of XPR1 promoted, while silencing of XPR1 inhibited the nuclear 


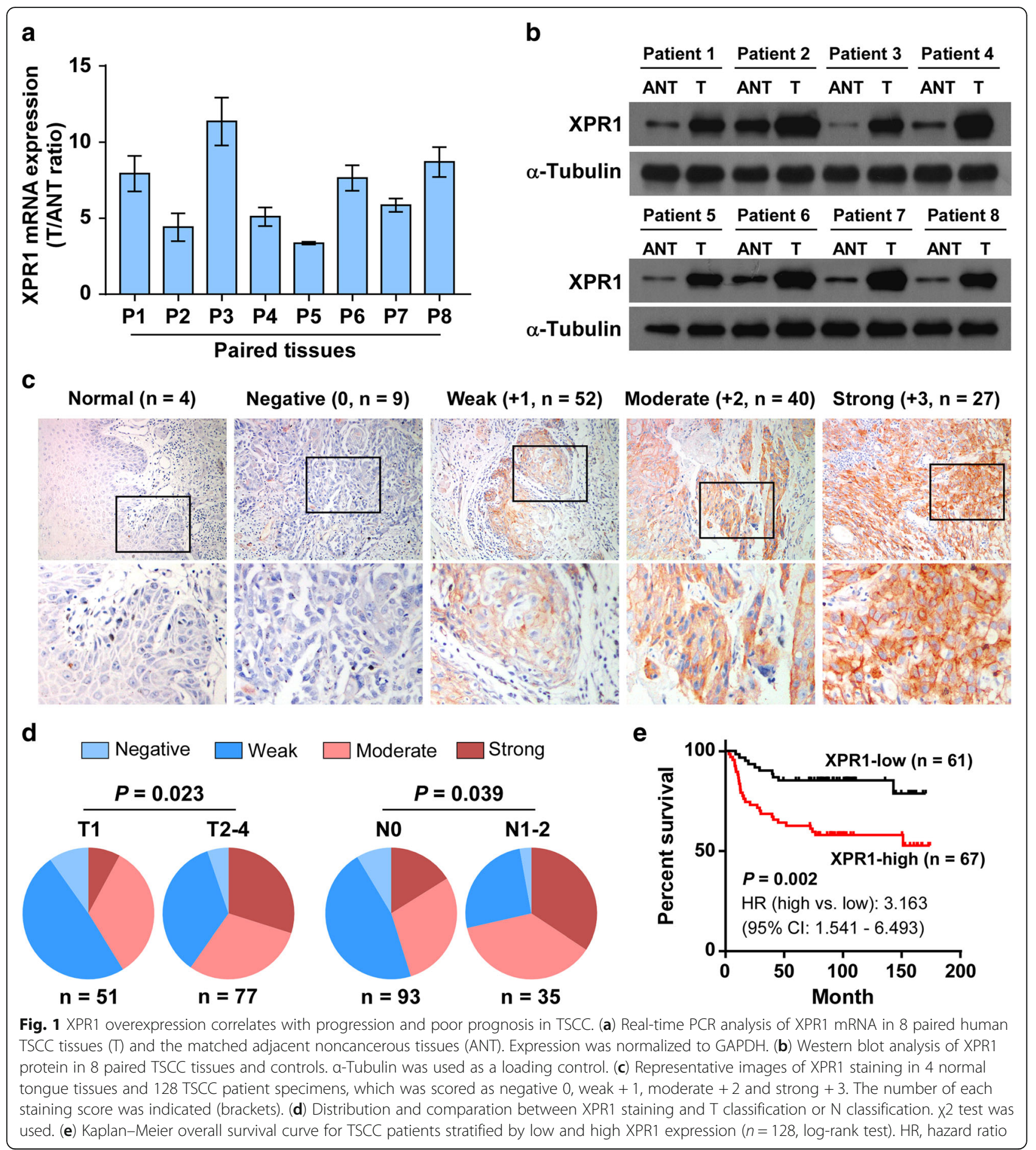

translocation of NF-kB/p65 in TSCC cells (Fig. $5 f$ and g). Moreover, real-time PCR analysis revealed that XPR1 regulted the expression of typical NF- $\mathrm{kB}$ downstream genes (Fig. 5h), which was further confirmed by IHC staining in xenografts (Fig. 5i). Thus, our findings suggest that XPR1 activates NF- $\mathrm{BB}$ signaling by increasing cAMP levels and subsequent PKA-mediated phosphorylation of NF-kB.
NF-KB activation was responsible for XPR1-mediated oncogenic effects

We then investigated whether NF- $\mathrm{kB}$ activation was essential for the oncogenic effects by XPR1. We inhibited NF- $\mathrm{BB}$ activity in XPR1-overexpressing SCC25 and CAL-27 cells by transfection of I $\mathrm{kB}-\alpha-$ mut plasmid which is non-degradative and restricts the p65/p50 complex in the cytoplasm, or treatment with NF- $\mathrm{kB}$ inhibitor 


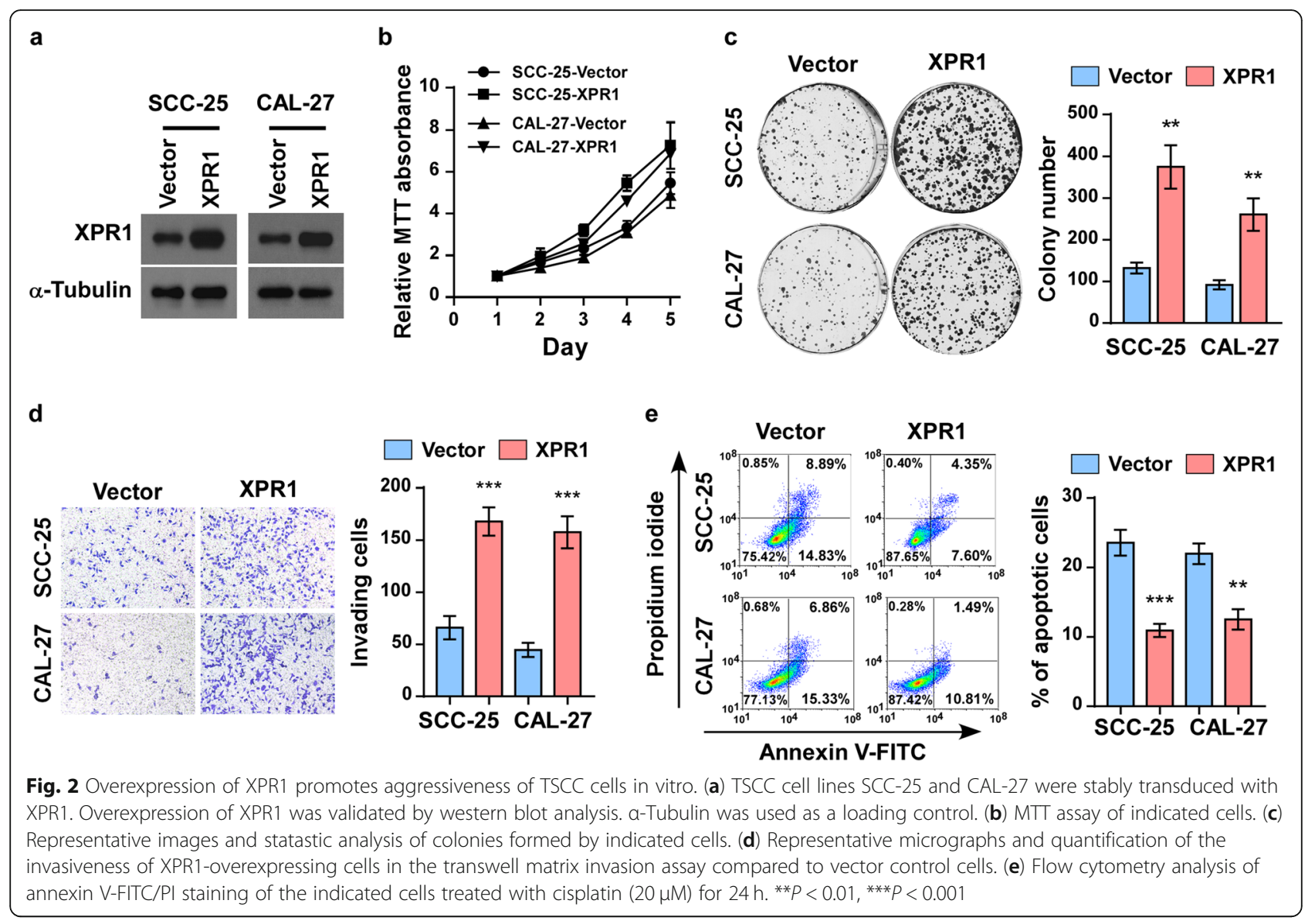

QNZ (EVP4593) to potently reduced the phosphorylation of NF- $\mathrm{KB}$ and inhibit NF- $\mathrm{KB}$ transcriptional activation. As expected, both $I_{k} B-\alpha-m u t$ overexpression and QNZ administration inhibited NF- $\mathrm{kB}$ activity (Additional file 1: Figure S3A), leading to robust suppression of the proliferation, invadsion, and anti-apoptosis capacities of TSCC cells (Fig. 6a-c). Overexpression of IкB- $\alpha$-mut significantly abrogated the promotive effects of XPR1 on the tumorigenecity of SCC-25 cells (Fig. 6d-f). Moreover, Ki67 IHC staining and TUNEL staining revealed that IкB- $\alpha$-mut reduced the proliferation index and promoted cell apoptosis in vivo (Fig. 6g). Thus, these results suggest that NF- $\mathrm{kB}$ activation is essential for XPR1-mediated aggressiveness in TSCC.

\section{Clinical relevance of the XPR1/NF-KB axis in TSCC}

Furthermore, we assessed whether the XPR $1 / N F-\kappa B$ axis identified in our study was clinically relevant. IHC staining of p65 and XPR1 was performed in the same cohort of 128 TSCC patient specimens as indicated in Fig. 6h. We found that high expression of XPR1 significantly correlated with the nuclear expression of p65 $(P=0.005$, Fig. 6h), which also predicted poor prognosis in TSCC patients $(P=0.010$, hazard ratio $(95 \% \mathrm{CI})=2.224(1.121-$ 4.414), Additional file 1: Figure S3B).

Taken together, our findings suggest that overexpression of XPR1 activates NF- $\mathrm{KB}$ signaling, leading to the malignant progression and poor clinical outcomes in human TSCC.

\section{Discussion}

XPR1, which was first identified as a cellular receptor for polytropic and xenotropic MLVs, has been shown to play a role in many pathophysiological processes. However, the clinical role of XPR1 in human cancers has not yet been characterized. Here, we demonstrate for the first time the potential role of XPR1 in TSCC progression and the potential value as a novel therapeutic target in human cancer. Clinical results show that high expression of XPR1 in TSCC patients correlated with a poor patient survival rate and more advanced stages. Overexpression of XPR1 induces TSCC cell aggressiveness with increased proliferation, invasion, and resistance to drug-induced apoptosis both in vitro and in vivo. Furthermore, XPR1 expression levels positively correlated with NF- $\mathrm{KB}$ signaling signatures, suggesting that XPR1 might be involved in regulation of the $N F-\kappa B$ signaling 

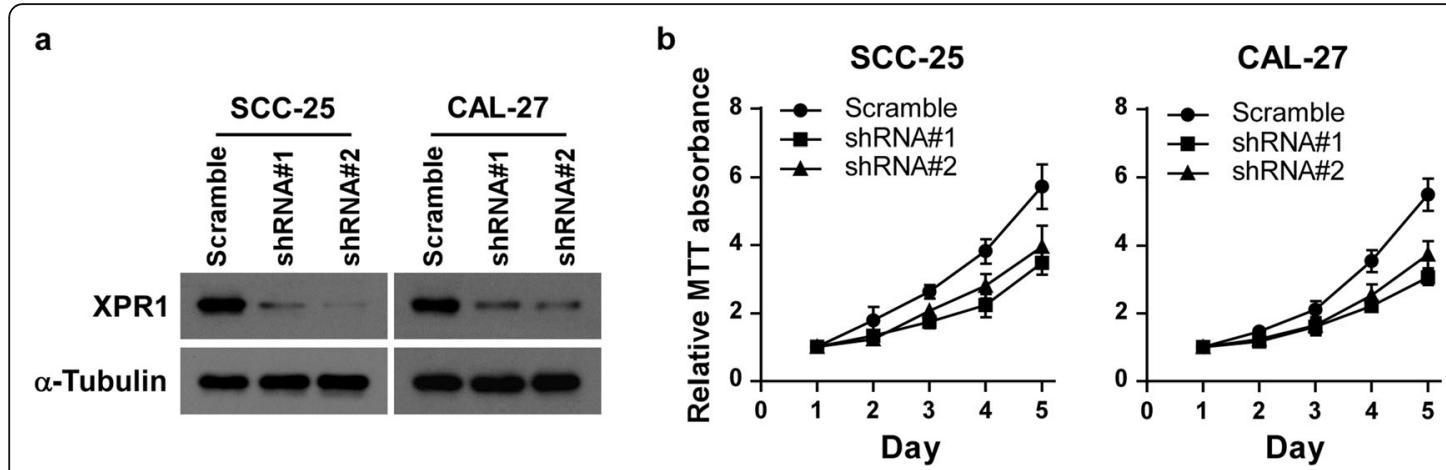
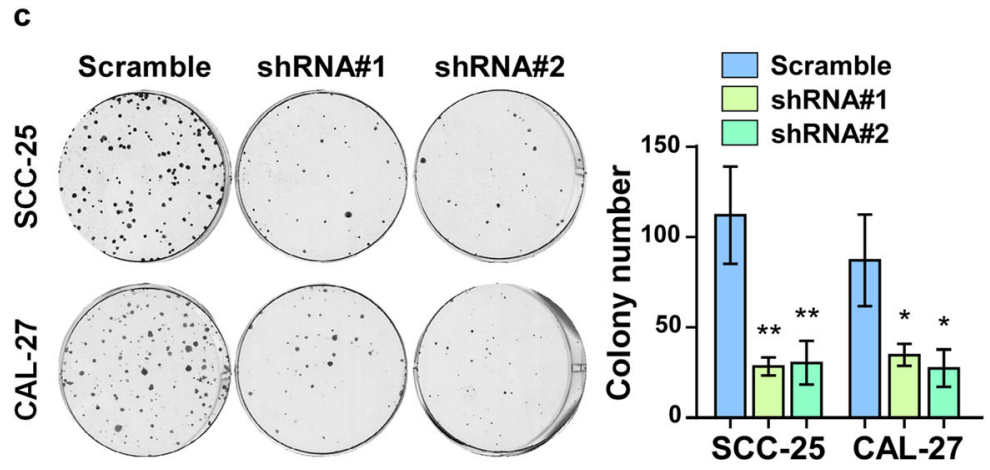

d

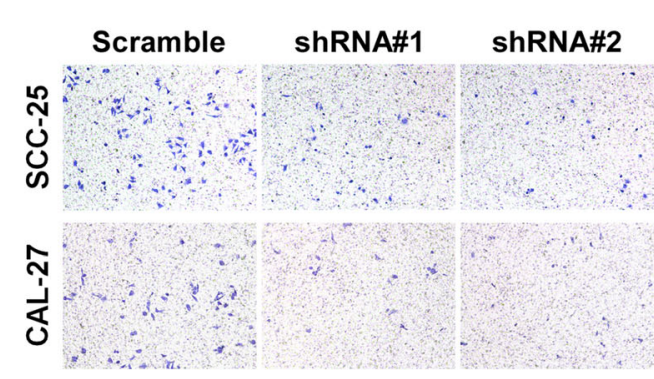

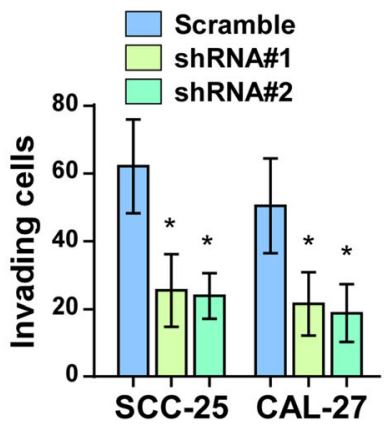
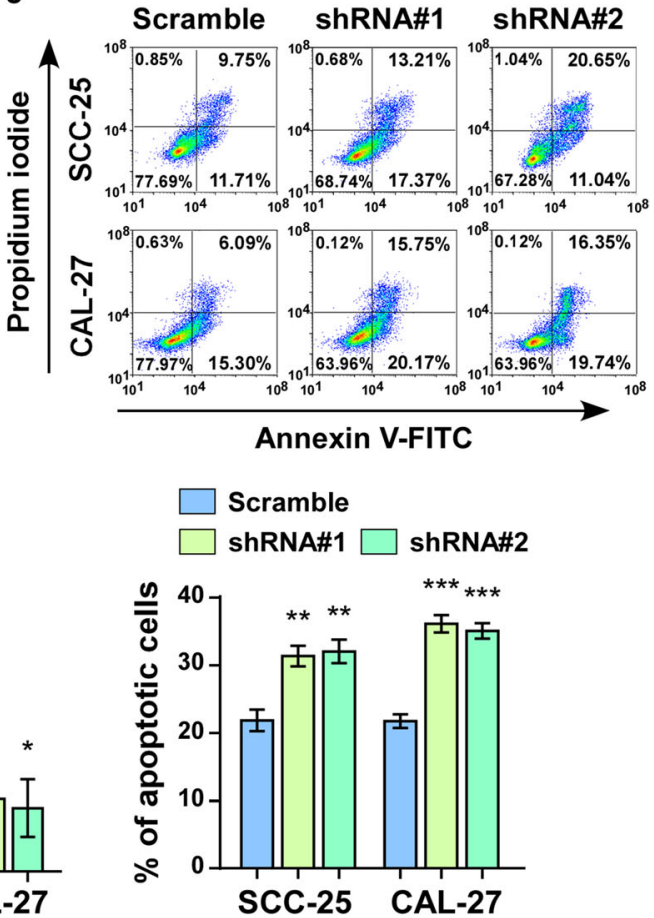

Fig. 3 Silencing of XPR1 reduced TSCC aggressiveness in vitro. (a) TSCC cell lines SCC-25 and CAL-27 were stably transduced with two XPR1 shRNAs. Knockdown of XPR1 was validated by western blot analysis. a-Tubulin was used as a loading control. (b) MTT assay of indicated cells. (c) Representative images and statastic analysis of colonies formed by indicated cells. (d) Representative micrographs and quantification of the invasiveness of XPR1-silenced cells in the transwell matrix invasion assay compared to scramble control cells. (e) Flow cytometry analysis of annexin V-FITC/PI staining of the indicated cells treated with cisplatin $(20 \mu \mathrm{M})$ for $24 \mathrm{~h}$. Quantification was shown below. ${ }^{*} P<0.05$, ${ }^{* *} P<0.01,{ }^{* * *} P<0.001$

pathway in TSCC. Consistently, the significant correlation detected between XPR1 levels and NF-kB signaling hyperactivation was confirmed in a cohort of human TSCC samples. Hence, this study defines a critical mechanism for XPR1-mediated constitutive activation of $\mathrm{NF}-\mathrm{KB}$ signaling, and identifies a new molecule with therapeutic potential for treating TSCC.

It has been acknowledged that the NF-кB pathway is an oncogenic signaling pathway that relevant to many aspects of tumorigenesis, including the control of apoptosis, cell cycle, invasion, differentiation, cell adhesion, cell migration, and angiogenesis [25]. To date, numerous researchers have confirmed that NF- $\mathrm{kB}$ signaling is constitutively activated in TSCC, and targeting NF- $\mathrm{kB}$ signaling contributes to characteristics of the malignant phenotype in TSCC, demonstrating the vital role of NF- $\mathrm{kB}$ signaling in TSCC progression. For instance, Lu KW et al. showed that Gyp, a component of Gynostemma pentaphyllum Makino, inhibits invasion and migration of human tongue SCC4 cells by downregulating related proteins RAS, NF- $\mathrm{B}, \mathrm{COX} 2$, ERK1/2, and MMP-9, finally reduces metastasis [26, 27]. Wang et al. have found that phosphorylated Ezrin (Tyr353) activates $\mathrm{NF}-\mathrm{kB}$, which results in enhanced metastasis of TSCC 


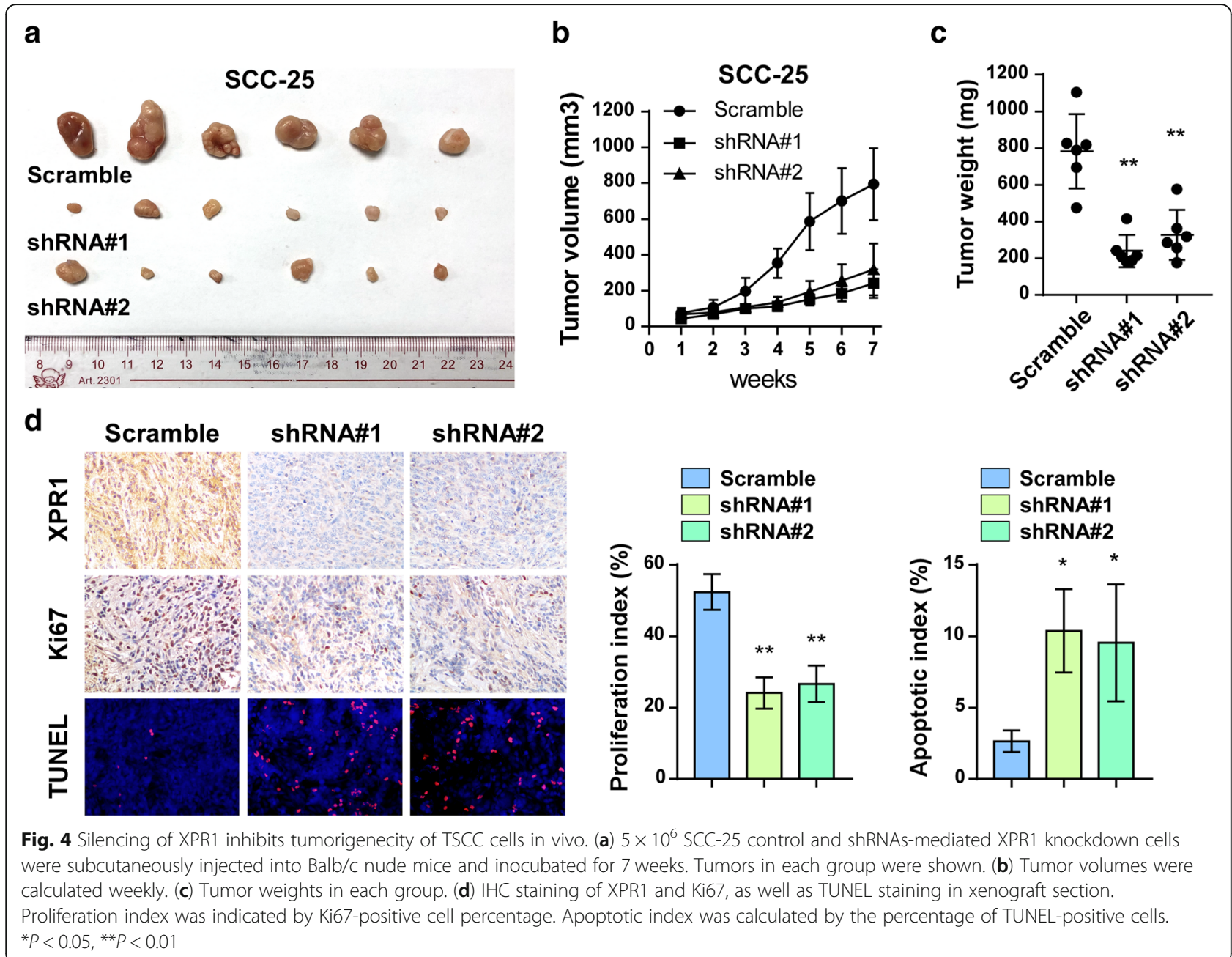

cells [28]. Activation of Ezrin and NF-kB is associated with cancer metastasis and poor patient prognosis [28]. Moreover, $\mathrm{Su}$ and colleagues showed that TRIM14, a member of the Tripartite Motif Containing (TRIM) family, was upregulated in TSCC and correlated with a more aggressive TSCC phenotype via activation of NF- $\mathrm{kB}$ signaling [29]. Collectively, dysregulation of NF- $\mathrm{BB}$ signaling has been well-documented in TSCC. Therefore, elucidating the underlying molecular mechanisms that regulate NF- $\mathrm{kB}$ signaling in TSCC could be important for the development of clinical TSCC therapies. Here, we show that overexpressing XPR1 enhanced NF- $\mathrm{kB}$ responsive luciferase activity and the expression of numerous well-characterized downstream genes of NF- $\mathrm{KB}$ signaling, whereas silencing of XPR1 had the opposite effects. This suggested that XPR1 might contribute to NF- $k B$ activation in TSCC.

Acting as a G-protein-coupled receptor, XPR1 was found to promote cAMP production [15]. Consistently, we found that XPR1 increased the intracellular concentration of cAMP. Thus, XPR1 activated PKA to phosphorylate p63 at ser276 site, leading to nuclear translocation and transcriptional activation of NF- $\mathrm{kB}$. These findings unveil a mechanism for XPR1-mediated NF- $\kappa \mathrm{B}$ activation. Interestingly, Miguel et al. showed that the transcription of mouse $X p r 1$ was significantly upregulated along with the $\mathrm{NF}-\mathrm{kB}$ activator $I k k \beta$, whereas the NF- $\kappa B$ inhibitor $I \kappa b \alpha$ was downregulated during disease progression [20]. The opposite results were observed in the mouse model in which $C D 40$ expression was silenced [24]. This finding indicates that expression levels of XPR1 are consistent with the activation of NF- $\mathrm{KB}$ signaling. Furthermore, XPR1 has also been reported to be overexpressed in human atherosclerotic plaques and localized in the perivascular adipose tissue, suggesting its relationship with chronic inflammation. In this study, we found the high expression of XPR1 could promote the abnormal activation of the NF- $\kappa B$ pathway. However, the mechanism of XPR1 upregulation in TSCC needs to be explored 

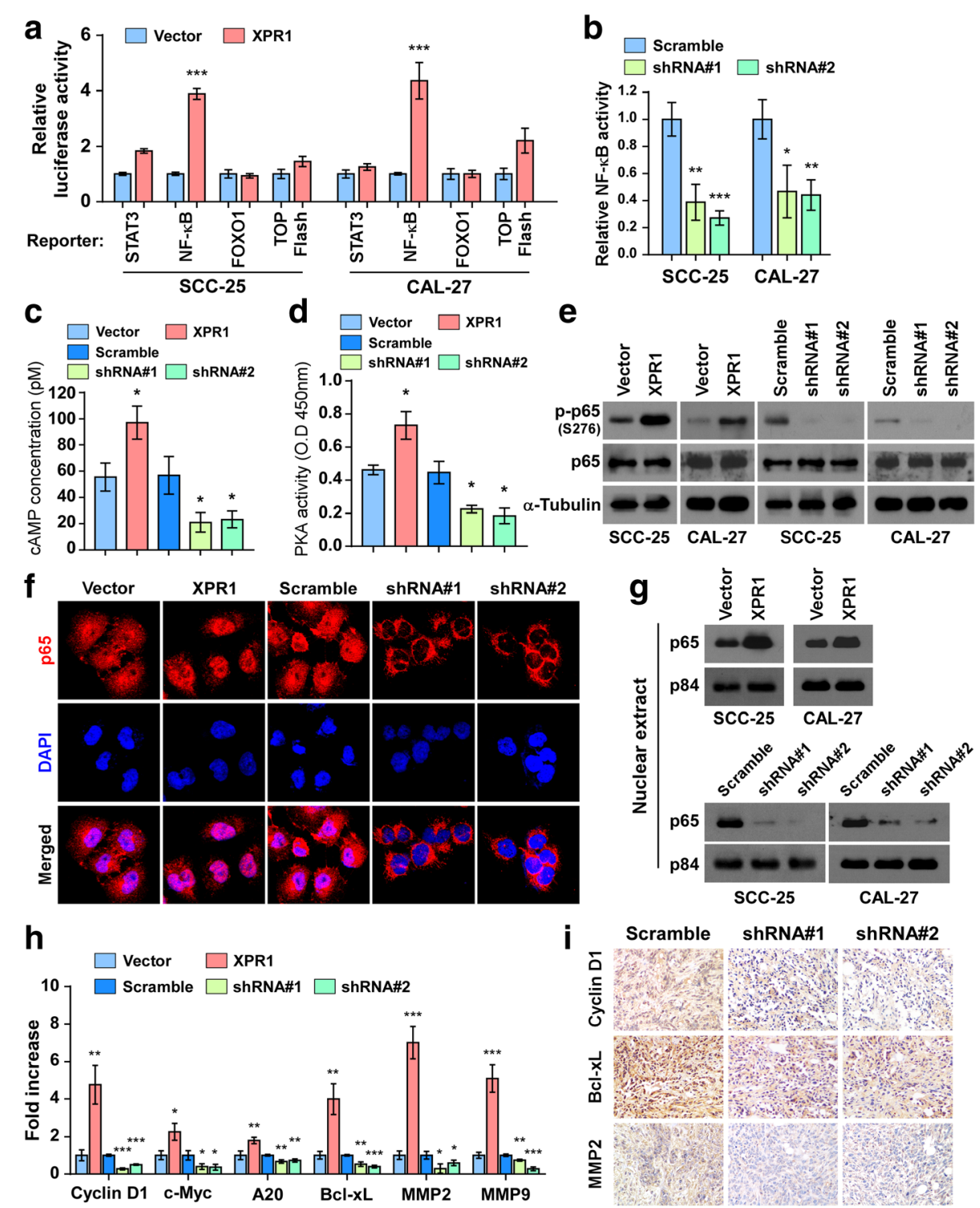

Fig. 5 XPR1 activates NF-kB signaling. (a) Luciferase reporter assays of STAT3, NF-KB, FOXO1 and TOP Flash reporters. (b) Relative NF-kB activity in XPR1-silenced cells and controls. (c) ELISA analysis was performed to determine the intracellular CAMP concentration in the lystates of SCC-25 with altered expression of XPR1. (d) Activity of PKA in indicated cells was measured by absorbance at $405 \mathrm{~nm}$. (e) Western blot analysis of p-p65S276, p65 and a-Tubulin in indicated cells. (f) Fluorescence immunostaining of the p65 location in indicated cells. (g) Nuclear fractions were isolated, and p65 expression was examined by immunoblotting. p84 was used as a nuclear protein marker. (h) Relative expression of NF-KB downstream genes was analyzed by real-time PCR. (i) IHC staining of Cyclin D1, BCl-xL and MMP2 in xenograft section. ${ }^{*} P<0.05$, ${ }^{* *} P<0.01,{ }^{* * *} P<0.001$

further. According to Miguel's group, non-canonical $\mathrm{NF}-\mathrm{KB}$ signaling might be relevant to the regulation of XPR1. Combined with our research, there may be a positive feedback interaction occurring between XPR1 and the NF-kB pathway.

Inorganic phosphate $(\mathrm{Pi})$, an essential nutrient to living organisms, is usually concentrated to promote malignant progression of tumors [30]. Accordingly, some inorganic phosphate transporters are upregulated in cancer and high serum $\mathrm{Pi}$ concentration correlates with morbidity and mortality of cancer [31]. Notably, recent evidence also indicates that a strong antiproliferative action of $\mathrm{Pi}$ in MDA-MB-231 cell line [32]. Moreover, high $\mathrm{Pi}$, cooperate with chemo-drug could be extremely toxic to particular cell types [33-35]. These studies implicate that $\mathrm{Pi}$ homeostasis might play a key role for cancer malignant progression [35]. Interestingly, XPR1 was recent found to promote export of Pi. In the current study, our results revealed that overexpression of XPR1 


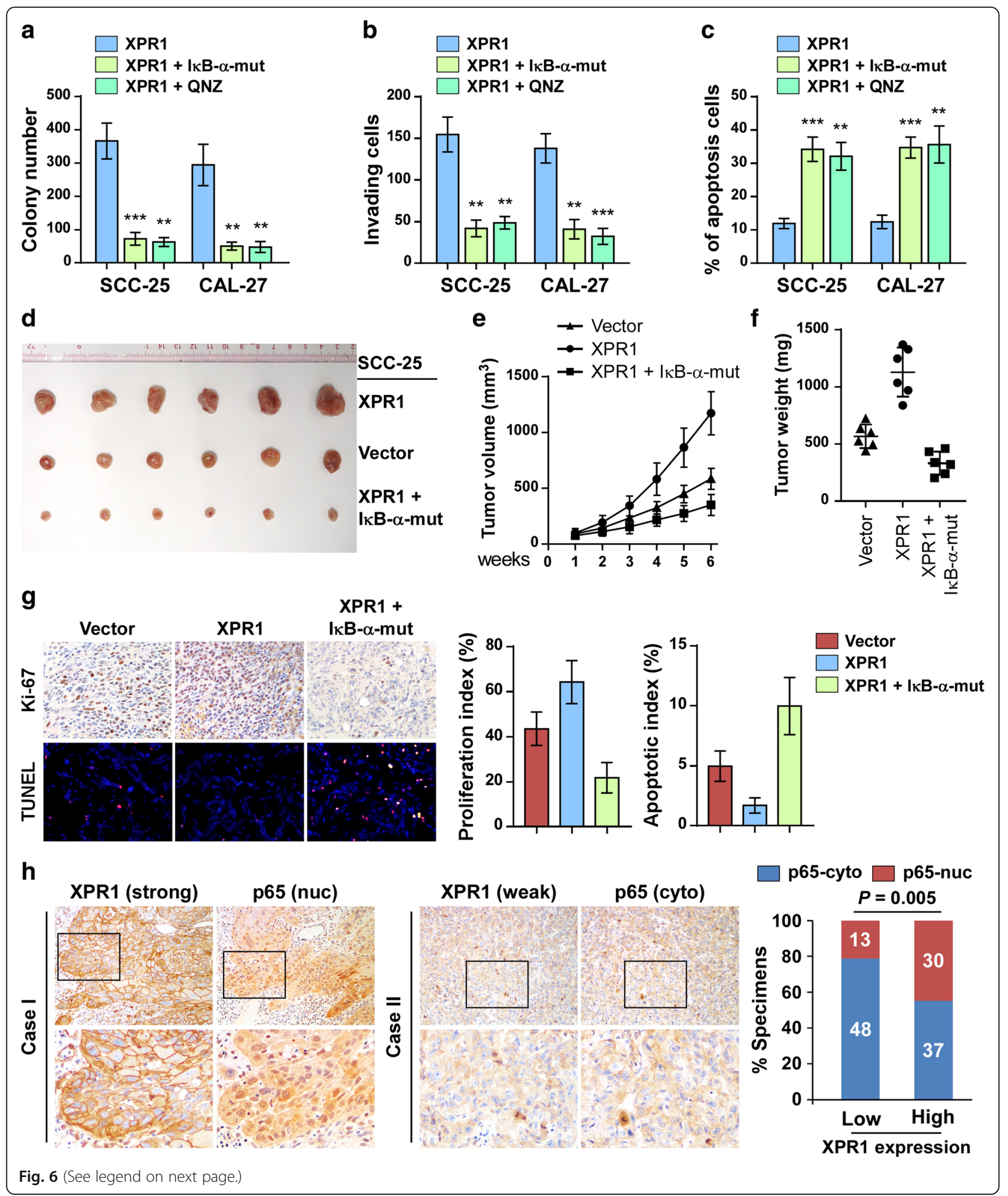


(See figure on previous page.)

Fig. 6 NF-KB activation mediates XPR1-mediated oncogenic effects. (a-c) XPR1-overexpressing SCC-25 and CAL-27 cells were transfected with IKBa-mut or treated with NF-KB inhibitor QNZ at $10 \mathrm{nM}$ for $24 \mathrm{~h}$, and then applied for colony formation assay (a), transwell penetration assay (b), and annnexin V/PI assay (c). (d) $5 \times 10^{6}$ vector control, XPR1-overexpressing, or XRP1 and IkB-a-mut-overexpressing SCC-25 cells were subcutaneously injected into Balb/c nude mice and inocubated for 6 weeks. Tumors in each group were shown. (e) Tumor volumes were calculated weekly. (f) Tumor weights in each group. (g) IHC staining of XPR1 and Ki67, as well as TUNEL staining in xenograft section. Proliferation index was indicated by Ki67-positive cell percentage. Apoptotic index was calculated by the percentage of TUNEL-positive cells. (h) Clinical relevace of XPR1 expression and NF-KB activation in patient specimens. Representative images of XPR1 and p65 IHC staining in 128 TSCC patient specimens. Correlation analysis revealed that high expression of XPR1 significantly associated with nuclear p65 expression. X2 test was used.

${ }^{*} P<0.01,{ }^{* * *} P<0.001$

significant inhibited the cytotoxic effects of cisplatin on TSCC cells. It thus could be postulated that XPR1 may reduce the $\mathrm{Pi}$ concentration to promote chemoresistance of TSCC cells; however, this hypothesis remains to be further investigated.

\section{Conclusion}

In summary, this study reveals that the elevated expression of XPR1 plays an important role in TSCC progression via activation of $\mathrm{NF}-\mathrm{kB}$ signaling. Our findings demonstrate for the first time a critical role for XPR1 in the progression of TSCC, and this work further elucidates the function of XPR1 in human disease. Understanding the precise roles of XPR1 in the pathogenesis and progression of TSCC and activation of the NF-kB signaling pathway will increase our knowledge of the biological basis of cancer, and might enable the development of novel therapeutic strategies against TSCC.

\section{Additional file}

Additional file 1: Table S1. Clinicopathological characteristics of 128 patient samples. Table S2. Univariate and multivariate analysis of factors associated with overall survival in 128 TSCC patients. Figure S1. (A) The XPR1 mRNA expression of TSCC tissues compared to normal controls by analyzing data set from The Cancer Genome Atlas (TCGA). (B) High expression of XPR1 significantly predicted poorer overall survival in patients with HNSC in TCGA data, as indicated by the human protein atlas program (https://www.proteinatlas.org/). (C) Number of specimen in different XPR1 staining score. Figure S2. (A and B) Flow cytometry analysis of annexin V-FITC/PI staining to determine the basal apoptosis rate of SCC-25 and CAL-27 cells with or without XPR1 overexpression (A) or knockdown (B). Figure S3. (A) Relative NF-kB reporter assay in indicated cells. (B) Kaplan-Meier overall survival curve for patients with p65-cytoplasma versus p65-nuclear. (PDF 659 kb)

\section{Abbreviations}

HNSC: Head and Neck Squamous Cell Carcinoma; MLV: Murine leukemia viruses; OSCC: Oral squamous cell carcinoma; PFBC: Primary familial brain calcification; TRIM: Tripartite Motif Containing; TSCC: Tongue squamous cell carcinoma; XPR1: Xenotropic and polytropic retrovirus receptor

\section{Acknowledgements}

Not applicable.

\section{Funding}

This work was supported by grants from the Science and Technology plan project of Guangdong Province (Nos. 2014A020212437, 2015A020210040, 2014A020212100); Nature Science Foundation of Guangdong Province (No.
2018A030313704); and the Research Project of Traditional Chinese Medicine Bureau of Guangdong Province (No. 20181065).

\section{Availability of data and materials}

The datasets are available from TCGA (http://cancergenome.nih.gov/) and human protein atlas program (https://www.proteinatlas.org/).

\section{Authors' contribution}

AKY and JNW designed the study; WCC, QLL, QMP, HYZ, XYF, FY and JNW conducted the experiments; WCC and HYZ performed the statistical analysis; WCC wrote the manuscript, and AKY and JNW helps to revise it. All authors read and approved the final manuscript.

\section{Ethics approval and consent to participate}

This study was approved by the Ethical Committee of the Sun Yat-sen University Cancer Center and informed consent was obtained from all patients. Additionally, in vivo experiments are in accordance with the protocols of Medicine Animal Care and Use committee.

\section{Consent for publication}

Not applicable.

\section{Competing interests}

The authors declare no conflict of interest.

\section{Publisher's Note}

Springer Nature remains neutral with regard to jurisdictional claims in published maps and institutional affiliations.

\section{Author details}

'Department of Head and Neck, Sun Yat-sen University Cancer Center, Guangzhou, Guangdong 510060, People's Republic of China. ${ }^{2}$ State Key Laboratory of Oncology in South China, Guangzhou, Guangdong 510060, People's Republic of China. ${ }^{3}$ Collaborative Innovation Center for Cancer Medicine, Guangzhou, Guangdong 510060, People's Republic of China. ${ }^{4}$ Guangzhou Yousheng Biotech Co., Ltd., Guangzhou, Guangdong 510060, People's Republic of China. ${ }^{5}$ Department of Oral and Maxillofacial Surgery, Guanghua School of Stomatology, Hospital of Stomatology, Institute of Stomatological Research, Sun Yat-sen University, Guangdong Provincial Key Laboratory of Stomatology, Guangzhou, Guangdong Province 510055, People's Republic of China.

Received: 7 October 2018 Accepted: 27 March 2019 Published online: 17 April 2019

\section{References}

1. Schwam ZG, Judson BL. Improved prognosis for patients with oral cavity squamous cell carcinoma: analysis of the National Cancer Database 19982006. Oral Oncol. 2016;52:45-51.

2. Warnakulasuriya S. Global epidemiology of oral and oropharyngeal cancer. Oral Oncol. 2009:45:309-16.

3. Cooper JS, Pajak TF, Forastiere AA, Jacobs J, Campbell BH, Saxman SB, Kish JA, Kim HE, Cmelak AJ, Rotman M, et al. Postoperative concurrent radiotherapy and chemotherapy for high-risk squamous-cell carcinoma of the head and neck. N Engl J Med. 2004;350:1937-44.

4. Tanvetyanon T, Padhya T, McCaffrey J, Kish JA, Deconti RC, Trotti A, Rao NG. Postoperative concurrent chemotherapy and radiotherapy for high-risk 
cutaneous squamous cell carcinoma of the head and neck. Head Neck. 2015:37:840-5.

5. Shah JP, Gil Z. Current concepts in management of oral cancer--surgery. Oral Oncol. 2009;45:394-401.

6. Blatt S, Voelxen N, Sagheb K, Pabst AM, Walenta S, Schroeder T, MuellerKlieser W, Ziebart T. Lactate as a predictive marker for tumor recurrence in patients with head and neck squamous cell carcinoma (HNSCC) post radiation: a prospective study over 15 years. Clin Oral Investig. 2016;20: 2097-104.

7. Bello IO, Soini Y, Salo T. Prognostic evaluation of oral tongue cancer: means, markers and perspectives (II). Oral Oncol. 2010;46:636-43.

8. Bello IO, Soini Y, Salo T. Prognostic evaluation of oral tongue cancer: means, markers and perspectives (I). Oral Oncol. 2010;46:630-5.

9. Leemans $\mathrm{CR}$, Braakhuis BJ, Brakenhoff RH. The molecular biology of head and neck cancer. Nat Rev Cancer. 2011;11:9-22.

10. Battini JL, Rasko JE, Miller AD. A human cell-surface receptor for xenotropic and polytropic murine leukemia viruses: possible role in G protein-coupled signal transduction. Proc Natl Acad Sci U S A. 1999;96:1385-90.

11. Tailor CS, Nouri A, Lee CG, Kozak C, Kabat D. Cloning and characterization of a cell surface receptor for xenotropic and polytropic murine leukemia viruses. Proc Natl Acad Sci U S A. 1999:96:927-32.

12. Yang YL, Guo L, Xu S, Holland CA, Kitamura T, Hunter K, Cunningham JM. Receptors for polytropic and xenotropic mouse leukaemia viruses encoded by a single gene at Rmc1. Nat Genet. 1999;21:216-9.

13. Spain BH, Koo D, Ramakrishnan M, Dzudzor B, Colicelli J. Truncated forms of a novel yeast protein suppress the lethality of a $\mathrm{G}$ protein alpha subunit deficiency by interacting with the beta subunit. J Biol Chem. 1995;270: 25435-44

14. Dorsam RT, Gutkind JS. G-protein-coupled receptors and cancer. Nat Rev Cancer. 2007;7:79-94.

15. Vaughan AE, Mendoza R, Aranda R, Battini JL, Miller AD. Xpr1 is an atypical G-protein-coupled receptor that mediates xenotropic and polytropic murine retrovirus neurotoxicity. J Virol. 2012;86:1661-9.

16. Stefanovic A, Arpat AB, Bligny R, Gout E, Vidoudez C, Bensimon M, Poirier Y. Over-expression of $\mathrm{PHO} 1$ in Arabidopsis leaves reveals its role in mediating phosphate efflux. Plant J. 2011;66:689-99.

17. Hurlimann HC, Pinson B, Stadler-Waibel M, Zeeman SC, Freimoser FM. The SPX domain of the yeast low-affinity phosphate transporter Pho90 regulates transport activity. EMBO Rep. 2009;10:1003-8.

18. Hurlimann HC, Stadler-Waibel M, Werner TP, Freimoser FM. Pho91 is a vacuolar phosphate transporter that regulates phosphate and polyphosphate metabolism in Saccharomyces cerevisiae. Mol Biol Cell. 2007; 18:4438-45.

19. Giovannini D, Touhami J, Charnet P, Sitbon M, Battini JL. Inorganic phosphate export by the retrovirus receptor XPR1 in metazoans. Cell Rep. 2013:3:1866-73.

20. Sharma P, Patntirapong S, Hann S, Hauschka PV. RANKL-RANK signaling regulates expression of xenotropic and polytropic virus receptor (XPR1) in osteoclasts. Biochem Biophys Res Commun. 2010;399:129-32.

21. Yao XP, Zhao M, Wang C, Guo XX, Su HZ, Dong EL, Chen HT, Lai JH, Liu YB, Wang $\mathrm{N}$, et al. Analysis of gene expression and functional characterization of XPR1: a pathogenic gene for primary familial brain calcification. Cell Tissue Res. 2017:370:267-73.

22. Anheim M, Lopez-Sanchez U, Giovannini D, Richard AC, Touhami J, N'Guyen L, Rudolf G, Thibault-Stoll A, Frebourg T, Hannequin D, et al. XPR1 mutations are a rare cause of primary familial brain calcification. J Neurol. 2016;263: 1559-64.

23. Legati A, Giovannini D, Nicolas G, Lopez-Sanchez U, Quintans B, Oliveira JR, Sears RL, Ramos EM, Spiteri E, Sobrido MJ, et al. Mutations in XPR1 cause primary familial brain calcification associated with altered phosphate export. Nat Genet. 2015;47:579-81.

24. Hueso M, De Ramon L, Navarro E, Ripoll E, Cruzado JM, Grinyo JM, Torras J. Silencing of CD40 in vivo reduces progression of experimental atherogenesis through an NF-kappaB/miR-125b axis and reveals new potential mediators in the pathogenesis of atherosclerosis. Atherosclerosis. 2016;255:80-9.

25. Karin M. Nuclear factor-kappaB in cancer development and progression. Nature. 2006;441:431-6.

26. Lu KW, Chen JC, Lai TY, Yang JS, Weng SW, Ma YS, Lu PJ, Weng JR, Chueh FS, Wood WG, et al. Gypenosides inhibits migration and invasion of human oral cancer SAS cells through the inhibition of matrix metalloproteinase-2 -9 and urokinase-plasminogen by ERK1/2 and NF-kappa B signaling pathways. Hum Exp Toxicol. 2011;30:406-15.

27. Lu KW, Tsai ML, Chen JC, Hsu SC, Hsia TC, Lin MW, Huang AC, Chang YH, Ip SW, Lu HF, et al. Gypenosides inhibited invasion and migration of human tongue cancer SCC4 cells through down-regulation of NFkappaB and matrix metalloproteinase-9. Anticancer Res. 2008;28:1093-9.

28. Wang Y, Lin Z, Sun L, Fan S, Huang Z, Zhang D, Yang Z, Li J, Chen W. Akt/ Ezrin Tyr353/NF-kappaB pathway regulates EGF-induced EMT and metastasis in tongue squamous cell carcinoma. Br J Cancer. 2014;110:695-705.

29. Su X, Wang J, Chen W, Li Z, Fu X, Yang A. Overexpression of TRIM14 promotes tongue squamous cell carcinoma aggressiveness by activating the NF-kappaB signaling pathway. Oncotarget. 2016;7:9939-50.

30. Lacerda-Abreu MA, Russo-Abrahao T, Monteiro RQ, Rumjanek FD, MeyerFernandes JR. Inorganic phosphate transporters in cancer: functions, molecular mechanisms and possible clinical applications. Biochim Biophys Acta Rev Cancer. 2018;1870:291-8.

31. Anderson JJ. Potential health concerns of dietary phosphorus: cancer, obesity, and hypertension. Ann N Y Acad Sci. 2013;1301:1-8.

32. Spina A, Sapio L, Esposito A, Di Maiolo F, Sorvillo L, Naviglio S. Inorganic phosphate as a novel signaling molecule with Antiproliferative action in MDA-MB-231 breast Cancer cells. BioRes Open Access. 2013;2:47-54.

33. Sapio L, Sorvillo L, Illiano M, Chiosi E, Spina A, Naviglio S. Inorganic phosphate prevents Erk1/2 and Stat3 activation and improves sensitivity to doxorubicin of MDA-MB-231 breast Cancer cells. Molecules. 2015;20:1591028.

34. Spina A, Sorvillo L, Di Maiolo F, Esposito A, D'Auria R, Di Gesto D, Chiosi E, Naviglio $S$. Inorganic phosphate enhances sensitivity of human osteosarcoma U2OS cells to doxorubicin via a p53-dependent pathway. J Cell Physiol. 2013;228:198-206.

35. Sapio L, Naviglio S. Inorganic phosphate in the development and treatment of cancer: a Janus Bifrons? World J Clin Oncol. 2015;6:198-201.
Ready to submit your research? Choose BMC and benefit from:

- fast, convenient online submission

- thorough peer review by experienced researchers in your field

- rapid publication on acceptance

- support for research data, including large and complex data types

- gold Open Access which fosters wider collaboration and increased citations

- maximum visibility for your research: over $100 \mathrm{M}$ website views per year

At BMC, research is always in progress.

Learn more biomedcentral.com/submissions 